\title{
The effects of different attentional loads on feature integration in the cerebral hemispheres
}

\author{
MIRJAM EGLIN \\ Institute for Behavioral Sciences, Eidgenössische Technische Hochschule (ETH), Zurich, Switzerland
}

\begin{abstract}
Two experiments were conducted to assess, in both visual fields, the occurrence of illusory conjunctions under two different attentional loads. To overload attention, verbal report of two digits was used as a primary task in Experiment 1 and a manual-response task indicating the orientation of two triangles was used in Experiment 2. An interaction between type of primary task and visual field on the efficiency of correctly recombining colors and shapes of colored letter stimuli was found. It was concluded that feature integration is achieved by the hemisphere of input, and that it can be selectively interfered with by processing demands made on hemisphere-specific attentional resources.
\end{abstract}

Several authors have proposed that the right hemisphere (RH) may play a special role in mediating attention by cerebral arousal and activation (Jutai, 1984), by sustaining attention (Dimond, 1979), by attending to both ipsiand contralateral stimuli (Geschwind, 1981; Heilman \& Van Den Abell, 1980), or by supporting the disengagement of attention from a current focus to move it to the left hemispace (Posner, Walker, Friedrick, \& Rafal, 1984). A large body of evidence documents attentional deficits with right-side lesions. Attention in the left hemisphere (LH), on the other hand, may be restricted to the ipsilateral hemispace (Geschwind, 1981; Heilman \& Van Den Abell, 1980) and may subserve selective or focal mechanisms (Dimond, 1979; Jutai, 1984). There is little experimental evidence, however, as to what specific attentional functions are actually supported by the LH. The experiments reported here were conducted to find out whether one specific function of focal attention, which serves the purpose of feature integration (Treisman \& Gelade, 1980), is especially associated with the LH.

According to feature-integration theory (Treisman \& Gelade, 1980), features on separable dimensions are registered automatically and in parallel. The subsequent recombination of separate features into multidimensional, localized objects requires serial processing with focal attention when there are features from several candidate objects and no conceptual constraints on how they combine. When attention is overloaded or diverted, incorrect recombinations of features (illusory conjunctions) are made (Treisman \& Schmidt, 1982).

Both serial processing and focal attention have been associated with left-hemisphere functions (Cohen, 1973; Di-

This research was supported by Swiss National Science Foundation Grant 1.545-0.82 and was conducted as part of a thesis submitted in partial fulfillment of the requirements for a $\mathrm{PhD}$ degree. Many thanks go to Anne Treisman, Dani Brandeis, and two anonymous reviewers for helpful comments on earlier versions of this manuscript. Correspondence concerning this article should be addressed to Mirjam Eglin, who is now at the School of Education, Tolman Hall, University of California, Berkeley, CA 94720. mond, 1979; Jutai, 1984; but see also Brand, Van Bekkum, Stumpel, \& Kroeze, 1983; Polich, 1982; Young \& Ellis, 1985), motivating several investigations on a possible LH specialization for feature integration. Indeed, serial search for a target defined by a conjunction of features was found to be relatively slower and more prone to error in the left visual field [LVF(RH)] than in the right visual field [RVF( $\mathrm{LH})]$, in contrast to search for a target that differed from the distractors by a single feature (Bolster, Harrington, \& Pribram, 1983). In the Bolster et al. study, stimulus displays of nine items each were presented for $100 \mathrm{msec}$, which was not sufficient time for a serial self-terminating search for the conjunctively defined target in the LVF(RH), resulting in a rather dramatic increase of errors from below $5 \%$ in all other conditions to about $30 \%$ in the conjunction condition. Thus, resource-limited (Norman \& Bobrow, 1975) conditions (due to limited exposure) were present, and the results indicate that for the kinds of stimuli used, serial search with focal attention is slower and more susceptible to disruption in the LVF(RH) than in the RVF(LH). In other studies, using evoked potential measures, greater amplitudes were recorded from the $\mathrm{LH}$ than from the $\mathrm{RH}$ for attendance to a target combination of color and word (Aine \& Harter, 1984) and for attendance to the combination of a relevant type of stimulus at a relevant location (Harter, Aine, \& Schroeder, 1982). The first finding could simply reflect a LH involvement for linguistic stimuli; the latter could not be replicated in a closely related paradigm (Hillyard \& Münte, 1984). Hillyard and Münte present evidence that evoked potential signs of feature selection clearly precede the signs of conjunction selection, but conjunction selection did not indicate any LH involvement. In summary, the evoked potential literature remains inconclusive and provides no unambiguous evidence for hemispheric asymmetry in feature integration.

Serial self-terminating search is difficult to demonstrate in divided-visual-field studies because of the necessary restrictions in exposure time. Resource limitations are 
almost inevitable. They result in a loss of localization information on individual features, giving rise to errors when these need to be recombined (Prinzmetal, 1981; Treisman \& Paterson, 1984; Treisman \& Schmidt, 1982). In divided-visual-field studies, this may produce inconclusive results. The greater RH load that was evident in Bolster et al.'s (1983) task might be a consequence of the type of stimuli used (colored shapes) rather than of the conjunction process.

Dynamic models of hemisphere functioning suggest that attentional capacity in the hemispheres can be selectively activated (Kinsbourne, 1975; Kinsbourne \& Hicks, 1978) or selectively loaded with specific tasks (Hellige, Cox, \& Litvac, 1979). The strongest claim for complete hemispheric independence of resource supplies was advanced by Friedman and Polson (1981). They suggest that the hemispheres comprise a system of two separate resource pools that are always activated to the same degree. To the extent that one hemisphere's capacity is employed in a task, that same amount of capacity is set free in the other hemisphere. Resources in the hemispheres may be further differentiated (Friedman, 1986). If there is independence of hemispheric resources, and if these can be selectively tapped by appropriate tasks, then the type of attentional load produced by a given task may play a crucial role in generating lateral asymmetry.

Two experiments were run to assess the occurrence of incorrect feature recombinations in both visual fields. These conjunction errors provide a means for studying the effects of different types of attentional load on the integrative function of attention in the hemispheres. In the first experiment, the primary task was to verbally report two digits before reporting colored letter stimuli. Speech production in right-handed subjects is controlled by the LH. The primary report task therefore was designed to draw more on left- than on right-hemisphere resources.

\section{EXPERIMENT 1}

\section{Method}

Subjects. Seven female and 12 male volunteers, with a mean age of 27.2 years, served as subjects. All were right-handed, as assessed by the Edinburgh Handedness Inventory for Laterality (Oldfield, 1971, translated into German), and all had normal or corrected-to-normal vision.

Apparatus and Stimuli. The stimuli were presented in an Electronic Developments three-field tachistoscope in a darkened room. The viewing distance for the stimuli was $50.8 \mathrm{~cm}$. Each stimulus card showed a column of three colored uppercase letters followed by two black digits. The three letters for each card were chosen from a set of five letters-I, $\mathrm{N}, \mathrm{O}, \mathrm{S}$, and X-and from a set of five colors-yellow, green, pink, blue, and brown. Each letter subtended a visual angle of $0.56^{\circ}$ horizontally and $0.79^{\circ}$ vertically. All letters could be turned upside-down without change. The letters were drawn by hand using colored inks and a Rotring (Art. 5495, $7 \mathrm{~mm}$ ) stencil. The whole letter configuration subtended a visual angle of $0.56^{\circ} \times 3.04^{\circ}$ and appeared $2.59^{\circ}$ of visual angle to the right or left of a black central fixation dot. Each color and letter appeared equally often in each position. Each of the different color-letter combinations appeared two to four times in each position. The two black digits were chosen from the set $1,6,8$, and 9. Each digit subtended a visual angle of $0.68^{\circ}$ horizontally and $0.45^{\circ}$ vertically. They were made from Alfac 2405 letterpress. The complete column of letters and digits subtended a visual angle of $5.06^{\circ}$ vertically.
Thirty test cards and 10 practice cards were made, half of them for the RVF and half for the LVF in each case. A noise mask consisting of equal numbers of randomly arranged black or white 2-mm squares (see Treisman \& Schmidt, 1982) with a black fixation dot in the center was used.

Procedure. The set of 30 cards was shuffled and shown to each subject four times, the second and fourth times turned upside down. Examples of the stimulus displays were shown to each subject and they were instructed first to report both digits and subsequently to report as many of the colors and letter shapes as they could remember. They were told to be as accurate as possible on the digits and preferably to report objects (e.g., "red X") and not single shapes or colors.

The experimenter gave a verbal "ready" signal and pressed a button to initiate a trial consisting of the following sequence: a black fixation dot appeared for approximately $1 \mathrm{sec}$, followed by the stimulus display for a variable duration, after which the noise mask appeared and remained on until the next trial was initiated. The exposure duration for the stimulus cards was initially set at $250 \mathrm{msec}$ for each subject and then adjusted according to the following procedure: If at least one color and one shape were reported correctly on seven successive trials, exposure duration was decreased by $50 \mathrm{msec}$; if a color or shape that was not on the card was reported on a trial or less than one color and one shape was reported on two consecutive trials, exposure duration was increased by $50 \mathrm{msec}$, but never above the maximum of $250 \mathrm{msec}$. If subjects made a mistake on the digits, that trial was discarded and was run once more at the end of the experiment.

\section{Results and Discussion}

Two subjects were excluded from the analysis because their error rates for reporting the digits were excessively high (88\% and $74 \%$ ). For the remaining 17 subjects the mean error rate on reporting the digits was $21 \%$ (ranging from $0 \%$ to $43 \%$ ). The mean number of valid trials was $\mathbf{4 9}$ for each visual field. The mean exposure duration was $241 \mathrm{msec}$. Responses were scored in the following way (also see Treisman \& Schmidt, 1982). The report of an individual color or shape (e.g., "pink" or "S") was scored as a single feature; an incorrectly perceived feature either conjoined with a correct or an incorrect feature (e.g., "blue S" or "yellow I" to the stimuli "pink I, brown O') was scored as a feature error; two correctly perceived features that were incorrectly recombined (e.g., "pink O" to the above stimuli) was scored as a conjunction error; and two correctly perceived features in the correct recombination (e.g., "pink I') was scored as a correct item. The "total features correct" category includes single correct features as well as correct features conjoined with either a correct or an incorrect feature. The mean numbers of the different types of response are given in Table 1.

Conjunction errors were significantly more frequent than feature errors $[t(16)=4.23, p<.001]$, indicating that they represented correctly perceived features that were incorrectly recombined rather than misperceived features that happened to be present on the same card (Treisman \& Schmidt, 1982). Within-subject comparisons of the different types of responses yielded the following effects: Conjunction errors were significantly more frequent in the RVF(LH) than in the LVF(RH) $[t(16)=3.20$, $p<.01]$, as was the total number of features reported correctly $[t(16)=2.42, p<.03]$. No other comparisons were significant [all $t \mathrm{~s}(16)<1.10, p>.1]$.

There was a general RVF(LH) advantage on the total number of correct features reported, as was to be expected 
Table 1

Mean Number Per Trial of Different Types of Responses in Both Visual Fields (Experiment 1)

\begin{tabular}{lcc}
\hline & LVF(RH) & RVF(LH) \\
\hline Single feature wrong & 0.06 & 0.05 \\
Single feature correct & 0.57 & 0.54 \\
Feature errors & 0.10 & 0.11 \\
Conjunction errors & 0.22 & $0.31^{*}$ \\
Correct items & 0.43 & 0.45 \\
Total features correct & 1.97 & $2.17 \dagger$ \\
\hline${ }^{*} p<.01 \quad \dagger p<.03$. & &
\end{tabular}

with letter stimuli (Beaumont, 1982). The excess of correct features reported in the $\operatorname{RVF}(\mathrm{LH})(.20)$ almost exactly equaled the surplus of incorrectly recombined features reported in this visual field $(.18$, i.e., twice the surplus of conjunction errors). The high rate of incorrect feature recombinations could thus be caused by different reporting strategies. Theoretically, subjects could have continued longer with reporting items on each trial in the RVF(LH), resulting in a loss of conjunction information and an increased rate of conjunction errors toward the end of their reports. However, the majority of conjunction errors in both visual fields were made on the first or only item reported $[75 \%$ in the $\mathrm{RVF}(\mathrm{LH})$ and $72 \%$ in the LVF(RH)], making it unlikely that lengthy reports determined the pattern of results in the RVF(LH). Similarly, reporting strategies in the visual fields could have differed with respect to how likely the colors and letter shapes were to be reported conjoined rather than separately. However, since the proportion of correctly perceived features reported conjoined was very similar in both visual fields $[0.73$ in the $\operatorname{LVF}(\mathrm{RH})$ and 0.75 in the RVF(LH); $t(16)<1]$, the excess of conjunction errors in the RVF(LH) can be attributed neither to lengthy reports nor to a tendency to report features conjoined rather than separately. Only the efficiency of correctly combining the correctly perceived features seems to have been selectively impaired in the RVF(LH). Indeed, the proportion of conjunction errors to all conjunctions of correct features $(=$ correct items plus conjunction errors) was significantly higher in the RVF(LH) than in the LVF(RH) $[t(16)=$ 2.36, $p<.03$ ] (see Figure 1).

Due to the long exposure durations, a contribution of eye movements to the RVF advantage in the total number of features reported correctly cannot be completely excluded. However, eye movements cannot explain why incorrect, but not correct, recombinations of features were more frequent in the RVF. In addition, the 4 subjects with the shortest exposure durations (mean $220 \mathrm{msec}$ ) did not differ from those with the longest ones (mean $250 \mathrm{msec}$ ) on the critical measure of the porportion of conjunction errors to all conjunctions of correct features, as tested by a two-way analysis of variance (ANOVA) (exposure $x$ visual field) [both $F_{\mathrm{s}}(1,6)<1.66, p>.10$ ].

To conclude, the verbal report task seems to have competed for the resources required to preserve and/or utilize the localization information on the individual features.
The fact that this competition for resources interfered more with RVF(LH) than with LVF(RH) conjoining suggests a certain independence of the hemispheres. Some extra capacity that supported the correct recombination of colors and letter shapes must have been available in the RH that was not accessible to the LH.

A second experiment was conducted to find out whether the excess of conjunction errors in the RVF $(\mathrm{LH})$ could be reduced by choosing a primary task with fewer resource demands on the LH. Experiment 2 differed from Experiment 1 only with respect to the primary task. The digits were replaced by shapes (triangles), and subjects were required to indicate their orientation by keypresses. Thus, neither the stimuli nor the response requirements of the primary task were specifically associated with $\mathrm{LH}$ functions.

\section{EXPERIMENT 2}

\section{Method}

Subjects. Five female and 7 male volunteers, with a mean age of 27.2 years, served as subjects ( 5 of them had also participated in the previous experiment). They were right-handed (Oldfield, 1971) and had normal or corrected-to-normal vision.

Apparatus and Stimuli. The stimulus cards were the same as those used in Experiment 1, except that, on each card, both digits were replaced by two black triangles. These triangles had equal side lengths of $0.45^{\circ}$ of visual angle. They pointed either to the right or to the left. On half the cards, the top and bottom triangles pointed in the same direction; on the other half, they pointed in different directions. To control for potentially different effects of the mask over the two visual fields, the mask used was the same as that used in Experiment 1.

The subjects had four response keys, two for the index and middle fingers of each hand for stimuli in the corresponding ipsilateral visual field. This response-key arrangement preserves what Wickens, Mountford, and Schreiner (1981) have termed taskhemispheric integrity: the stimuli are presented to the same hemisphere that initiates the motor response.

Procedure. The procedure was the same as that used in Experiment 1, except that the primary task now was to indicate whether the triangles pointed in the same or in different directions. All subjects used the index finger to indicate "same" and the middle finger to indicate "different." If a subject made a mistake on the triangles, that trial was discarded without being repeated.

\section{Results and Discussion}

The mean error rate on the triangle task was $16 \%$, leaving a mean number of 52 valid trials for the LVF(RH) and 50 for the RVF(LH). The mean exposure duration was $236 \mathrm{msec}$. The mean number of conjunction and feature errors per trial, along with other types of responses, are shown in Table 2. Conjunction errors were significantly more frequent than feature errors $[t(11)=6.11$, $p<.001$, again indicating that they represent correctly perceived features that were incorrectly recombined. The comparison of both visual fields showed that more unconjoined features were reported in the LVF(RH) than in the RVF(LH) $[t(11)=2.91, p<.02]$, whereas more correct items $[t(11)=4.50, p<.001]$ and more cor- 
rect features overall $[t(11)=4.93, p<.001]$ were reported in the RVF(LH) than in the LVF(RH). Conjunction errors and also conjunction minus feature errors were equally frequent in both visual fields, as were feature errors [all $t s(11)<1$ ].

Again, reporting the colored letters was more efficient in the RVF( $(\mathrm{LH})$ than in the $\operatorname{LVF}(\mathrm{RH})$, as evidenced by the higher rate of correct features reported in this visual field. Unlike the first experiment, there were now more correct items in the RVF(LH) than in the LVF(RH). A surplus of correct items might be expected because more features were perceived correctly in the RVF(LH) than in the LVF $(\mathrm{RH})$ and therefore more of them could be conjoined. However, the proportion of correct items to the total of correct features was also higher in the RVF(LH) (.26) than in the $\operatorname{LVF}(\mathrm{RH})(.20)[t(11)=3.52$, $p<.005$ ], indicating that correctly perceived features were more likely to be correctly conjoined in the RVF(LH) than in the LVF(RH). This suggests that conjunction information was available more readily in the RVF(LH) than in the LVF(RH). The reduced localization information available in the LVF(RH) became apparent in a surplus of unconjoined correct features rather than in a pronounced increase of incorrectly conjoined features in this visual field. Although the proportion of conjunction errors to all conjunctions of correct features was still higher in the LVF(RH) than in the RVF(LH), the difference was not significant $[t(11)=1.70, p>.1]$ (see Figure 1).

The performance of the 5 subjects who had participated in the previous experiment was compared with that of the naive subjects by means of a two-way ANOVA (subjects $x$ visual field). There was no difference between groups [both $F \mathrm{~s}(1,10)<1.22, p\rangle .10$ ]. Also, the four subjects with the shortest exposures (mean $216 \mathrm{msec}$ ) did not differ from those with the longest exposures (mean $247 \mathrm{msec}$ ) on the proportion of conjunction errors to all conjunctions of correct features, as tested by a two-way ANOVA (exposure $\times$ visual field) (both $F \mathrm{~s}<1$ ). It is therefore unlikely that the long exposure durations or the reuse of 5 subjects in Experiment 2 biased the findings.

When the results of both experiments are compared, an interaction between primary tasks and the proportions of conjunction errors to all conjunctions of correct features is apparent. A two-way ANOVA (visual field $x$ primary task) on these proportions of conjunction errors

Table 2

Mean Number Per Trial of Different Types of Responses in Both Visual Fields (Experiment 2)

\begin{tabular}{lcc}
\hline & LVF(RH) & RVF(LH) \\
\hline Single feature wrong & 0.09 & 0.07 \\
Single feature correct & 0.79 & $0.66^{*}$ \\
Feature errors & 0.17 & 0.13 \\
Conjunction errors & 0.36 & 0.35 \\
Correct items & 0.56 & $0.78 \dagger$ \\
Total features correct & 2.80 & $3.05 \dagger$ \\
\hline
\end{tabular}

${ }^{*} p<.02 . \quad \neq p<.001$.

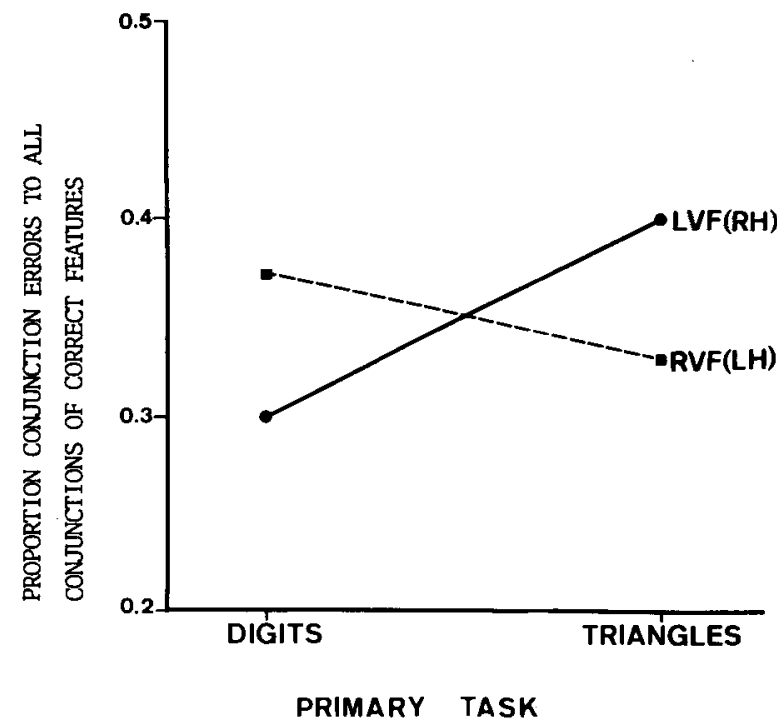

Figure 1. The mean proportion of conjunction errors to all conjunctions of correct features in both visual fields in Experiments 1 and 2.

confirmed this effect $[F(1,27)=8.05, p<.01]$, which is illustrated in Figure 1.

\section{GENERAL DISCUSSION}

The results of both experiments confirm that illusory conjunctions are directly related to the amount of attention that is available (Treisman \& Gelade, 1980; Treisman \& Schmidt, 1982). It is noteworthy that the specific attentional loads used, which involved specific structures (vocal/manual) and/or resources (verbal/visuospatial), affected such an early operation as feature integration. It seems that extra resources were needed in the $\mathbf{L H}$ for the vocal/verbal (the digit task) relative to those needed for the visuospatial/manual (the triangle task) primary task, as selectively reflected in an impaired efficiency of correctly conjoining features. Similarly, conjunction errors in the $\mathbf{L F}(\mathbf{R H})$ reflected the different task demands of the visuospatial/manual and the verbal/vocal task in this hemisphere.

The interaction between primary tasks and the proportion of correct conjunctions in each hemisphere suggests that the integration of features by focal attention is achieved by the hemisphere of input. It follows that the conjunction process cannot be lateralized to one hemisphere. Serial, attended processing is carried out in both hemispheres. Its efficiency is dependent upon the amount of resources available in each hemisphere.

The fact that feature integration can be selectively influenced in each visual field by manipulation of the nature of the primary task supports the assumption that hemispheric resources are controlled separately (Friedman \& Polson, 1981). Dual-task methodology is used to demonstrate relative independence of hemispheric 
resources, as, for example, when performance on a visual verbal memory task can be traded only with $\mathrm{LH}$, and not with RH, performance in an auditory tone memory task (Herdman \& Friedman, 1985), or when a verbal memory load interferes with $\mathrm{LH}$, but not with $\mathrm{RH}$, performance in a dichotic listening task (Hellige \& Wong, 1983). For conclusions regarding the specificity of resources, it is important to study the performance tradeoffs between tasks by manipulating task priority (Friedman, 1986) or by varying the nature of one of the tasks, as in the present experiments. Both primary tasks interfered with feature conjoining in both hemispheres. This was to be expected, since the digits and triangles were initially perceived in the same hemisphere as the colored letters. More importantly, however, the manipulation of the relative resource demands of each primary task was selectively reflected in the proportion of correctly perceived features that could be correctly conjoined in each hemisphere.

How specific the type of attention involved in feature integration is has never been investigated experimentally (Prinzmetal, Presti, \& Posner, 1986). It may be specific to vision, in which case the response demands of the primary tasks used here (vocal/manual) may not have influenced the results. Remember that on each trial the primary task was completed before the colored letters were reported. The primary tasks therefore could interfere only with the perception of the colored letters and not with their report. Also remember that a change in asymmetry occurred only for the proportion of conjunction errors and not for the total correct features reported. Therefore, if perception of the colors and letter shapes is automatic in the present task (Treisman \& Gelade, 1980), feature conjoining may be the first and only perceptual processing stage at which there is competition for resources. All subsequent processing, which involves mainly verbally reporting the colored letters, either may be carried out after the primary task has been completed or may be functionally localized in the $\mathrm{LH}$, in which case the lateral asymmetry in the total features correct reflects interhemispheric transmission rather than hemispheric differences in processing efficiency. If there is functional localization, no changes in asymmetry can be produced by a concurrent task, since stimuli from both visual fields are equally affected (Moscovitch, 1986; Umiltà, Rizzolatti, Anzola, Luppino, \& Porro, 1985). Neither of these accounts permits any conclusions as to which elements of the primary tasks competed with the conjunction process.

The present results are special in the sense that the dependent measures were illusory conjunctions, which actually represent failures of attention. The tasks were designed to overload attention, and conjunction errors would not appear under less resource-limited conditions. However, due to restrictions in exposure duration, resource limitations are a common characteristic of divided-visual-field tasks. Other studies have shown that attention may mediate lateral asymmetries also for more complex or higher order processes, such as letter match- ing or lexical decisions (Chiarello, 1985; Schwartz \& Kirsner, 1982). It may be important to distinguish strongly lateralized tasks that may be localized in one hemisphere from weakly lateralized tasks that are performed in each hemisphere but with different processing efficiency. Concurrent tasks can produce changes in asymmetry for the latter but not for the former (Moscovitch, 1986). However, it seems that differential tradeoffs are obtained among a great variety of tasks (Friedman, 1986), and few tasks may be completely lateralized to one hemisphere. As a consequence, hemisphere-specific attentional resources may play a crucial role in generating lateral asymmetries. Experiments involving one specific type of stimuli or type of task may involve specific resource compositions and must remain inconclusive in this respect. As in the above experiments, results from a given task may not generalize to other types of stimuli or tasks.

In the present discussion, the operation of feature integration has been equated with a serial processing step. The idea that serial and parallel processing are associated with a specific information processing step may shed new light on a longstanding controversy concerning these two processing modes in the hemispheres. For example, although word encoding as a typical LH function has been found to rely on parallel processing of the component letters, in the RH, these letters are processed serially (e.g., Brand et al., 1983; Young \& Ellis, 1985). Serial processing is necessary when top-down constraints are insufficient to localize separate features (Treisman \& Gelade, 1980). Lexicality has been shown to function as such a constraint, perceptually unitizing single words (Prinzmetal \& Millis-Wright, 1984), and this effect may be especially pronounced in the language-specialized LH. Thus, serial/parallel processing may reflect task conditions (e.g., the need for feature integration) rather than a hard-wired processing mode.

\section{REFERENCES}

AINE, C. J., \& HARTER, M. R. (1984). Hemispheric differences in eventrelated potentials to STROOP stimuli: Attention and color/word processing. In R. Karrer, J. Cohen, \& P. Tueting (Eds.), Sixth International Conference on Event-Related Slow-Potentials of the Brain. New York: Academy of Sciences.

BEAUMONT, J. G. (1982). Divided visual field studies of cerebral organisation (pp. 57-86). London: Academic Press.

Bolster, R. B., Harrington, M. J., Pribram, K. H. (1983, February). Hemispheric processing of visual targets in multidimensional displays. Paper presented at the meeting of the International Neuropsychological Society, Mexico City.

Brand, N., Van BekKum, I., Stumpel, M., \& Kroeze, J. H. A. (1983). Word matching and lexical decisions: A visual half-field study. Brain \& Language, 18, 199-211.

Chiarello, C. (1985). Hemisphere dynamics in lexical access: Automatic and controlled processes. Brain \& Language, 26, 146-172.

CoHEN, G. (1973). Hemispheric differences in serial versus parallel processing. Journal of Experimental Psychology, 97, 349-356.

Dimond, S. J. (1979). Performance by split-brain humans on lateralized vigilance tasks. Cortex, 15, 43-50.

Friedman, A. (1986). Dividing attention between the hands and the mind. Journal of Clinical \& Experimental Neuropsychology, 8, 143.

Friedman, A., Polson, M. C. (1981). The hemispheres as indepen- 
dent resource-systems: Limited-capacity processing and cerebral specialization. Joumal of Experimental Psychology: Human Perception \& Performance, 7, 1031-1058.

Geschwind, N. (1981). The perverseness of the right hemisphere. Behavioral \& Brain Sciences, 4, 106-107.

HARTER, M. R., AINe, C., \& SCHRoEder, C. (1982). Hemispheric differences in the neural processing of stimulus location and type: Effects of selective attention on visual evoked potentials. Neuropsychologia, $20,421-438$.

Heilman, K. M., \& Van Den Abell, T. (1980). Right hemisphere dominance for attention: The mechanisms underlying hemispheric asymmetries of inattention (neglect). Neurology, 30, 327-330.

Heluge, J. B., CoX, P. J., \& LitvaC, L. (1979). Information processing in the cerebral hemispheres: Selective hemispheric activation and capacity limitations. Journal of Experimental Psychology: General, 108, 251-279.

Hellige, J. B., \& WoNG, T. M. (1983). Hemisphere-specific interference in dichotic listening: Task variables and individual differences. Journal of Experimental Psychology: General, 112, 218-239.

Herdman, C. M., \& Friedman, A. (1985). Multiple resources in divided attention: A cross-modal test of the independence of hemispheric resources. Journal of Experimental Psychology: Human Perception \& Performance, 11, 40-49.

HillyaRD, S. A., \& MüNTE, T. F. (1984). Selective attention to color and location: An analysis with event-related brain potentials. Perception \& Psychophysics, 36, 185-198.

JuTAI, J.W. (1984). Cerebral asymmetry and the psychophysiology of attention. International Journal of Psychophysiology, 1, 219-225.

KInsBourne, M. (1975). The mechanism of hemispheric control of the lateral gradient of attention. In P. M. A. Rabbit \& S. Dornic (Eds.), Attention and performance V (pp. 81-97). New York: Academic Press.

KInSBOURNe, M., \& Hicks, R. E. (1978). Functional cerebral space. A model for overflow, transfer and interference effects in human performance. In J. Requin (Ed.), Attention and performance VII (pp. 345362). Hillsdale, NJ: Erlbaum.

Moscovitch, M. (1986). Afferent and efferent models of visual perceptual asymmetries: Theoretical and empirical implications. Neuropsychologia, 24, 91-114.

Norman, D. A., Bobrow, D. G. (1975). On data-limited and resource-limited processes. Cognitive Psychology, 7, 44-64.
OLDFIELD, R. C. (1971). The assessment and analysis of handedness: The Edinburgh Inventory. Neuropsychologia, 9, 97-113.

Pouch, J. M. (1982). Hemispheric differences for visual search: Serial versus parallel processing revisited. Neuropsychologia, 20, 297-307.

Posner, M. I., Walker, J. A., Friedrich, F. J., \& RAfal, R. D. (1984). Effects of parietal injury on covert orienting of attention. Journal of Neuroscience, 4, 1863-1874.

Prinzmetal, W. (1981). Principles of feature integration in visual perception. Perception \& Psychophysics, 30, 330-340.

Prinzmetal, W., Millis-Wright, M. (1984). Cognitive and linguistic factors affect visual feature integration. Cognitive Psychology, 16, 305-340.

Prinzmetal, W., Presti, D. E., \& Posner, M. I. (1986). Does attention affect visual feature integration? Journal of Experimental Psychology: Human Perception \& Performance, 12, 361-369.

SCHWARTZ, S., \& KIRSNER, K. (1982). Laterality effects in visual information processing: Hemispheric specialisation or the orienting of attention? Quarterly Joumal of Experimental Psychology, 34A, 61-77.

Treisman, A. M., \& Gelade, G. (1980). A feature-integration theory of attention. Cognitive Psychology, 12, 97-136.

Treisman, A., \& Paterson, R. (1984). Emergent features, attention, and object perception. Journal of Experimental Psychology: Human Perception \& Performance, 10, 12-31.

Treisman, A., \& SchmidT, H. (1982). Illusory conjunctions in the perception of objects. Cognitive Psychology, 14, 107-141.

Umiltà, C., Rizzolatti, G., Anzola, G. P., Luppino, G., \& PorRo, C. (1985). Evidence of interhemispheric transmission in laterality effects. Neuropsychologia, 23, 203-213.

Wickens, C. D., Mountford, S. J., \& SChreIner, W. (1981). Multiple resources, task-hemispheric integrity, and individual differences in time-sharing. Human Factors, 23, 211-229.

Young, A. W., \& ElLIs, A. W. (1985). Different methods of lexical access for words presented in the left and right visual hemifields. Brain \& Language, 24, 326-358.

(Manuscript received July 25, 1986; revision accepted for publication January $21,1987$. 